\title{
Pathoclysis in aging human cerebral cortex: Evidence from in vivo MRI morphometry
}

\author{
NAFTALI RAZ, IVAN J. TORRES, and WESLEY D. SPENCER \\ Memphis State University, Memphis, Tennessee \\ and \\ JAMES D. ACKER \\ Baptist Memorial Hospital, Memphis, Tennessee
}

\begin{abstract}
Age-related differences in the cross-sectional area and volume of selected cortical regions were examined by using in vivo magnetic resonance morphometry. In two samples-healthy volunteers and patients with negative radiological findings-similar patterns of cortical aging emerged. The size of sampled regions of association cortices correlated negatively with age, whereas no significant correlations between the size of sensory cortices and age were found. In the first but not the second sample, some of the correlations were attenuated after statistical control for skull size and sex. Overall, small but consistent trends for leftward asymmetry of the white matter and rightward asymmetry of the gray matter were observed. The results of the reported studies support the notion of selective aging of cortical association areas accompanied by relative sparing of sensory cortices.
\end{abstract}

Aging is accompanied by significant changes in brain structure. However, neuroanatomical markers of senescence repeatedly found in postmortem investigations, computerized tomography, and magnetic resonance imaging (MRI) studies are nonspecific. These most robust findings consist of ventriculomegaly and shrinkage of the cerebral parenchyma, particularly of the gray matter (Freedman, Knoefel, Naeser, \& Levine, 1984; Jernigan et al., 1991; Jernigan, Press, \& Hesselink, 1990; Lim, Zipurski, Watts, \& Pfefferbaum, 1992; Miller, Alston, \& Corsellis, 1980; Schwartz et al., 1985). Such results notwithstanding, there are reasons to believe that cerebral aging may not be as uniform and nonspecific as has been implied. Since pathologists investigating the aged human brain are "often struck by the focal nature of ... cortical atrophy" (Katzman \& Terry, 1983, p. 35), it seems plausible that brain aging is, at least in its early stages, localized and selective.

Indeed, several postmortem investigations indicate that in addition to diffuse neuropathology, intensified regional cerebral atrophy may be observed in the apparently healthy elderly. Cortical areas believed to be especially susceptible to aging include the prefrontal, inferior parietal, and entorhinal cortices, as well as the hippocampal

\footnotetext{
We gratefully acknowledge the assistance of the technical personnel at both MRI sites. Administrative assistance by Cornelia Gates is greatly appreciated. This project was supported in part by Biomedical Research Support Grant S07-RR0-5366-26 and by the Center for Applied Psychological Research of the Department of Psychology, Memphis State University. Correspondence should be addressed to N. Raz, Department of Psychology, Memphis State University, Memphis, TN 38152 (e-mail: razn@memstvx1.bitnet).
}

formation (Arriagada \& Hyman, 1990; Brody, 1973; Haug, 1985; Lim, Zipursky, Murphy, \& Pfefferbaum, 1990; Mani, Lohr, \& Jeste, 1986; Scheibel, 1981; Terry, DeTeresa, \& Hansen, 1987). The results of neuroanatomical studies of primates indicate that the visual and motor cortices are virtually unaffected by aging (Tigges, Herndon, \& Peters, 1990; Vincent, Peters, \& Tigges, 1989), although some human postmortem data reveal age-related atrophy in the macular projection area of the striate cortex (Devaney \& Johnson, 1980). In general, neuropathological studies have revealed greater age-related deterioration in high-order association cortices and the hippocampus than in the primary sensory areas (Kemper, 1984). This type of selective vulnerability of brain tissue was dubbed pathoclysis by Vogt and Vogt (1951), who argued that some gray-matter structures (grisea) exhibited increased susceptibility to pathogens.

Although pathoclysis does not have to be limited to the gray-matter regions, the evidence of selective age-related changes in the white matter has been inconsistent. Agerelated changes in molecular properties of the hemispheric white matter in anterior temporal and prefrontal regions have been noticed (Raz, Millman, \& Sarpel, 1990; Wahlund et al., 1990). These changes, expressed as prolongation of proton relaxation times, imply altered myelination. Indeed, postmortem findings from the turn of the century suggest that in the aging brain, demyelination of the fibers underlying association areas may $\propto c-$ cur, while the projections to and from primary sensory areas may remain intact (Kaes, 1907, cited in Kemper, 1984). White-matter abnormalities are frequently detected on the MRI scans of normal elderly (Drayer, 1988), yet no age-related reduction in gross volume of the intra- 
hemispheric white matter has been observed in MRI (Jernigan et al., 1991).

In a recent neuroimaging study (Coffey et al., 1992), significant age-related shrinkage of the cerebral hemispheres, the frontal and the temporal lobes, and the hippocampal formation was observed. Coffey and colleagues also reported that age-related decreases were greater in the frontal lobes than in other brain regions. Although suggestive of pathoclytic cerebral changes, the conclusions of that study were limited by several factors. First, only regions known for age-dependent declines were selected for measurement; no areas expected to be spared by aging were considered. Second, the white and the gray matter were not differentiated when the target regions were delineated, and many structurally and functionally distinct areas were combined into grossly defined lobes.

In the studies reported here, our goal was to explore regional differences in the aging cerebral cortex, and to document age-related pathoclysis in vivo. To increase the specificity of the measures, gray-and white-matter areas were estimated separately, and efforts were made to dissociate the regions of the cortex that are sensitive to aging from those that are not. On the basis of the postmortem evidence reviewed above, we hypothesized that in addition to age-dependent expansion of the cerebral ventricles, a distinct pattern of age-related differences would be observed. Specifically, the more dedicated the area is to the primary processing of modality-specific information, the fewer age-related changes it should show. Thus, we expected that polymodal association cortices (e.g., dorsolateral prefrontal cortex, DLPFC, and inferior parietal lobule, IPL) and the hippocampal formation would be found to shrink with age, whereas we hypothesized that the primary somatosensory cortex and the primary and secondary visual areas would show only minimal agerelated reduction.

To test these hypotheses, we obtained volumetric and planimetric measures from MRI scans in two samples. The first consisted of a carefully selected group of healthy volunteers spanning a wide age range (Study 1). The second was a sample of clinical MRI scans acquired from persons who presented with neurological complaints that led to negative diagnostic findings (Study 2). Because of the differences in the appearance of several key structures on MRI scans acquired with different scanners under different combinations of parameters, the samples were not combined.

\section{STUDY 1}

\section{Method}

\section{Subjects}

The sample consisted of 29 healthy volunteers (self-reported righthanders). All participants were interviewed by the senior author (N.R.). They denied a history of psychiatric and neurological disorders, alcoholism, diabetes, or hypertension-the conditions that are known to produce neuromorphological changes and to disrupt brain structure and cognitive functioning of the elderly (Peres, Kane,
\& Aronson, 1973; Skullerud, 1985; White, Cartwright, CornoniHuntley, \& Brock, 1986). At the time of the study, none of the subjects was on a psychoactive medication. All elderly subjects were either retirees who lived independently or gainfully employed individuals. All subjects scored at least 29 out of 30 points on the Mini-Mental Status Examination (MMSE; Folstein, Folstein, \& McHugh, 1975). The mean age in this sample that included 17 males and 12 females was 43.8 years $(S D=21.5)$, with a range of $18-78$ years. On the average, the subjects had 15.0 years of formal education. All participants signed an informed consent form and were thoroughly debriefed concerning the objectives of the study.

The majority of the subjects $(N=26)$ underwent on-line measurement of regional spin-lattice relaxation times $\left(T_{1}\right)$. The results of these measurements have been reported previously (Raz et al., 1990). Cognitive measures were administered to all 29 subjects to assess the relationship between cerebral and cognitive aging in a sample of healthy volunteers. The results of this part of the study are discussed elsewhere (Raz et al., in press).

\section{Apparatus and Procedure}

Imaging was performed on a Beta-3000 0.3T permanent-magnet scanner (Fonar Corp., Melville, NY) at the University of Health Sciences/Chicago Medical School (North Chicago). Each subject's head was aligned along the midline, using a light beam focused on the glabella. This position was maintained by placing a foam-padded restraint frame around the subject's head. Two spin-echo imaging sequences were performed. Nine sagittal slices were obtained at echo time (TE) $=28 \mathrm{msec}$ and repetition time (TR) $=600 \mathrm{msec}$, number of excitations $(\mathrm{NEX})=3$, slice thickness $(\mathrm{t})=4.2 \mathrm{~mm}$, and interslice interval $(\mathrm{I})=6.0 \mathrm{~mm}$. The position of the coronal slices was determined with reference to the middle of the pituitary (slice 0 ) as observed on the midsagittal slice. Coronal slices located rostrally and caudally to that slice received ascending numbers with a negative or a positive sign, respectively. A total of 17-21 coronal images (depending on the cranial size) were acquired with $\mathrm{TE}=$ $28 \mathrm{msec}, \mathrm{TR}=1,800 \mathrm{msec}, \mathrm{NEX}=2, \mathrm{t}=6.6 \mathrm{~mm}$, and $\mathrm{I}=8.6 \mathrm{~mm}$.

\section{Computer-Assisted Image Analysis}

Digital MRI planimetry was performed with the use of JAVA software (Jandel Scientific, San Rafael, CA). The MRI films were positioned on a light table and digitized frame by frame with a video camera and a frame-grabber board. The images were printed with the mean window setting of $W=633.21(S D=245.77)$ and mean level setting of $L=396.25(S D=101.29)$. Neither parameter correlated with age ( $r=-.07$ and -.13 , respectively, both $n . s$.). The areas of interest (AOIs) were outlined on the screen by trained operators (W.D.S. and I.J.T.) and were digitally enhanced. Areas of the contrast-enhanced structures were computed with the JAVA software. Whenever multiple slices were available, the volumes of regions of interest (ROIs) were calculated by multiplying the sum of AOI areas by the interslice interval. In defining and measuring the ROIs, we used the following rules.

Skull size. To estimate the skull size, the area bound by the in ner perimeter of the skull, a straight line connecting the glabella and the rostral tip of the clivus, and a horizontal straight line connecting the caudal edge of the clivus to the back of the skull was outlined as depicted in Figure 1a.

Dorsolateral prefrontal cortex. The cross-sectional areas of the DLPFC and the adjacent frontal-lobe white matter were measured on the first slice rostral to the genu of the corpus callosum (Figure 1b). The area of the DLPFC including the superior and the middle gyri and pars triangularis of the inferior frontal gyrus was outlined on this slice as depicted in Figure 1b. The outlined areas of the neocortex corresponded approximately to Brodmann's areas 8, 9, and 46 and regions F04, F06, and F07 in H. Damasio and A. R. Damasio's (1989) nomenclature. The lower (ventral) border 

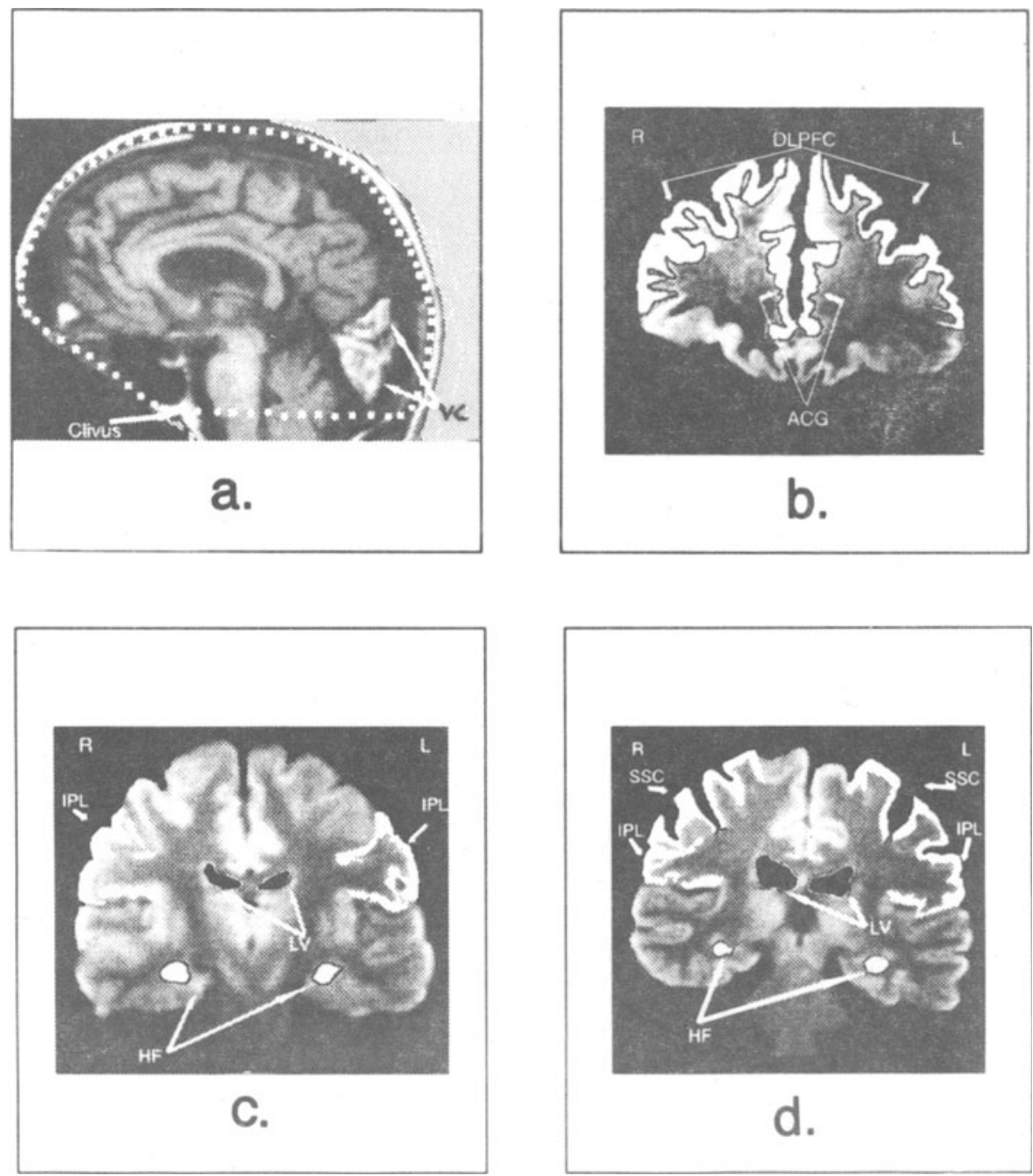

Figure 1. (a) Regions of interest measured on the midsagittal MRI slice: cranial cross-sectional area (dotted line), and visual cortical areas with adjacent white matter (VC). (b) Regions of interest highlighted on coronal slice +5: dorsolateral prefrontal cortex (DLPFC), anterior cingulate gyrus (ACG). (c) Regions of interest measured on the coronal slice 0: hippocampal formation (HF), inferior parietal lobule (IPL), lateral ventricles (LV). (d) Regions of interest measured on slice - 1: hippocampal formation (HF), primary somatosensory cortex (SSC), inferior parietal lobule (IPL), lateral ventricles (LV).

of the DLPFC was defined by the anterior ramus of the lateral sulcus. The upper boundary of the DLPFC was defined by the most dorsomedial point of the white matter in the superior frontal gyrus.

Anterior cingulate gyrus (ACG). The ACG (Figure 1b) was measured on the same slice as was the DLPFC. The upper and lower boundaries were defined by the superior and inferior cingulate sulci.

Prefrontal white matter. The total white-matter area was outlined on the same slice where the DLPFC and ACG were measured, complementing the previously mentioned gray-matter regions.

Hippocampal formation. The volume of the hippocampal formation was estimated from coronal slices 0 and -1 as depicted in Figures $1 \mathrm{c}$ and $1 \mathrm{~d}$. The hippocampus proper could not be discerned reliably. The hippocampal formation was demarcated rostrally by the amygdala, laterally and ventrolaterally by the temporal-lobe white matter, and dorsomedially by the ambient cistern. To define the medial boundary of the hippocampal formation, a vertical line was drawn from the dorsomedial edge of the formation to cut the subiculum at that level.

Postcentral gyrus (primary somatosensory cortex). The crosssectional area of the primary sensory cortex (postcentral gyrus, Brodmann's 1-3, post-Rolandic part of the Damasios' region F08) was estimated from slice -1 (Figure 1d). To locate the inferior boundary of the primary somatosensory cortex (the postcentral sulcus), a line was drawn along the dorsal surface of the lateral ventricle and extended to the surface of the cortex. The postcentral sulcus was the first sulcus located at or below the intersection of the line with the cortical ribbon. The lower bank of the paracentral sulcus served as the upper boundary of the primary somatosensory cortex.

Inferior parietal lobule. The volume of the IPL (Brodmann's 40, the Damasios' region P01) was estimated from slices 0 and - 1 (Figures $1 \mathrm{c}$ and $1 \mathrm{~d})$. The lower boundary of the IPL was the Sylvian fissure, and its upper boundary was the postcentral sulcus (deter- 
mined above as the lower boundary of the primary somatosensory cortex).

Parietal white matter. This was outlined on slices 0 and -1 as the area complementary to the primary somatosensory cortex and the IPL, bound ventrally by the temporal stem.

Lateral ventricles. The volume of the lateral ventricles was estimated from all coronal slices on which they appeared; the choroid plexus was included.

Visual areas (primary and secondary). The cross-section of the cortex and the white matter was outlined on the midsagittal slice following the parieto-occipital sulcus as presented in Figure 1a. This ROI included mesial aspects of both supra- and infracalcarine areas (Brodmann's 17-19, the Damasios' O01 and 002).

The identification of all regions and structures was verified against the atlases of correlative MRI neuroanatomy (Daniels, Naughton, \& Naidich, 1987; Montemurro \& Bruni, 1980) and general neuroanatomy atlases (DeArmond, Fusco, \& Dewex, 1976; Nieuwenhuys, Voogd, \& Huijzen, 1988). The operators were unaware of the subjects' age or sex.

Interrater reliability of morphometric estimates was assessed with the intraclass correlations formula for random raters (ICC[2], Shrout $\&$ Fleiss, 1979). The computations were based on two operators (I.J.T. and W.D.S.) and 12 randomly selected subjects. The results of the reliability analysis indicate that the measures were highly reliable: intraclass correlations ranged from .89 (postcentral gyrus) to .98 (midskull area).

\section{Results and Discussion}

The zero-order correlations among the ROI measures, skull size, and age are presented in Table 1. The pattern of zero-order correlations suggests selective age-related shrinkage of cortical structures. However, the size of many ROIs correlated positively with the skull size, which, in turn, negatively correlated with age. Skull size is established in childhood or, at the latest, in early adolescence (Finlay \& Richardson, 1990) and presumably cannot reflect adult age-related changes. Therefore, age differences in cranial capacity imply cohort effects, secular trends, or sampling bias rather than the influence of senescence per se. To ensure the validity of conclusions regarding brain aging, one ought to control for the variation in cranial size. The same logic is applicable to sex difference in brain morphology, for females usually have smaller crania than males do.
To estimate the effects of age, sex, and the hemispheric location on the size of the selected brain structures, the derived morphometric estimates were submitted to a mixed linear model analysis. In this model, the effect of the hemisphere (left vs. right, a repeated measures factor), sex (a categorical variable), and age (a continuous variable) were adjusted for the differences in skull size. To adjust the lateral differences to the mean skull size rather than to a zero, the deviation scores (the mean skull size minus the individual measure) were used. In all models, interactions between the continuous variables (age and skull size) and the grouping factor (sex) were tested first, to ascertain the homogeneity of regression. If found nonsignificant, the interaction terms were removed, and reduced models were analyzed.

The models for all dependent variables except DLPFC area were successfully reduced. The results of the analyses are presented in Table 2 (descriptive statistics and laterality effects) and Table 3 (age and sex effects).

\section{Hemispheric Differences}

Several lateral differences were observed in the brains examined in this sample. The hippocampal formation was significantly larger on the right. For all other gray-matter regions, cortical and subcortical, trends toward rightward asymmetry were observed, although none of these trends reached statistical significance after adjustment for skull size, age, and sex. The prefrontal and parietal whitematter volumes were greater on the left than on the right, although the trend was statistically significant only for the parietal lobes. The left lateral ventricle was significantly larger than the right.

\section{Age Differences}

Most of the age trends suggested by the zero-order correlations in Table 1 were attenuated by controlling for skull size and sex. The only significant age effect that remained after the adjustment was age-related enlargement of the lateral ventricles.

The model for DLPFC contained a significant age $x$ skull size $X$ sex interaction $[F(1,21)=5.87, p<.025]$.

Table 1

Correlations Among Age and the Brain Structures

\begin{tabular}{lcccccccccc}
\hline Structure & DLPFC & ACG & IPL & SSC & VC & HF & FWM & PWM & Lat V & Age \\
\hline Skull & $.48 \dagger$ & .22 & $.42^{*}$ & -.26 & $.65 \ddagger$ & .14 & $.63 \ddagger$ & $.57 \ddagger$ & -.07 & $-.41^{*}$ \\
DLPFC & & .32 & $.45^{*}$ & -.15 & $.38^{*}$ & .16 & $.44^{*}$ & $.43^{*}$ & -.02 & $-.43^{*}$ \\
ACG & & & $.36^{*}$ & -.12 & .32 & .05 & -.07 & -.10 & -.03 & -.18 \\
IPL & & & & .01 & .11 & $.36^{*}$ & .33 & .32 & -.16 & $-.42^{*}$ \\
SSC & & & & & .04 & .19 & .20 & .21 & $-.47 \dagger$ & -.19 \\
VC & & & & & & .11 & $.49 \dagger$ & $.37^{*}$ & -.07 & -.28 \\
HF & & & & & & & .25 & -.02 & -.06 & -.03 \\
FWM & & & & & & & & $.51 \dagger$ & -.25 & $-.36^{*}$ \\
PWM & & & & & & & & & -.18 & -.31 \\
Lat V & & & & & & & & & & $.60 \ddagger$ \\
\hline
\end{tabular}

Note-DLPFC, dorsolateral prefrontal cortex; ACG, anterior cingulate gyrus; IPL, inferior parietal lobule; SSC, somatosensory cortex; VC, visual cortex; HF, hippocampal formation; FWM and PWM, frontal and parietal white matter; Lat V, lateral ventricles. ${ }^{*} p<.05 . \quad \nmid p<.01 . \quad \neq p<.001$. 
Table 2

Volumes and Areas of Cortical Structures

\begin{tabular}{|c|c|c|c|c|c|}
\hline \multirow[b]{3}{*}{ Structure } & \multicolumn{4}{|c|}{ Size (by Side) } & \multirow{3}{*}{$\begin{array}{c}\begin{array}{c}\text { Left-Right } \\
\text { Comparison }\end{array} \\
\frac{F}{}\end{array}$} \\
\hline & \multicolumn{2}{|c|}{ Left } & \multicolumn{2}{|c|}{ Right } & \\
\hline & $M$ & $S D$ & $M$ & $S D$ & \\
\hline DLPFC $\left(\mathrm{cm}^{2}\right)$ & 6.88 & .75 & 7.34 & .99 & $3.91^{2}$ \\
\hline $\operatorname{ACG}\left(\mathrm{cm}^{2}\right)$ & 2.60 & .43 & 2.70 & .36 & 2.23 \\
\hline IPL $\left(\mathrm{cm}^{3}\right)$ & 11.98 & 1.68 & 12.43 & 1.66 & 2.28 \\
\hline PCG $\left(\mathrm{cm}^{3}\right)$ & 3.51 & .66 & 3.66 & .99 & .35 \\
\hline$F W M\left(\mathrm{~cm}^{3}\right)$ & 12.97 & 2.68 & 12.24 & 3.21 & 1.48 \\
\hline PWM $\left(\mathrm{cm}^{3}\right)$ & 34.15 & 4.10 & 32.18 & 3.81 & $27.56 \ddagger$ \\
\hline $\mathrm{HF}\left(\mathrm{cm}^{3}\right)$ & 1.12 & .20 & 1.25 & .20 & $6.99^{*}$ \\
\hline \multirow[t]{2}{*}{ Lat $V\left(\mathrm{~cm}^{3}\right)$} & 15.60 & 9.90 & 13.44 & 10.28 & $7.33 \dagger$ \\
\hline & & \multicolumn{2}{|c|}{$M$} & $S D$ & \\
\hline \multirow{2}{*}{\multicolumn{2}{|c|}{$\begin{array}{l}\text { Midskull area }\left(\mathrm{cm}^{2}\right) \\
\text { Visual areas }\left(\mathrm{cm}^{2}\right)\end{array}$}} & \multirow{2}{*}{\multicolumn{2}{|c|}{$\begin{array}{r}203.77 \\
12.02\end{array}$}} & \multicolumn{2}{|c|}{24.57} \\
\hline & & & & & 87 \\
\hline
\end{tabular}

${ }^{1}$ Effects after adjustment for skull size and sex. ${ }^{2}$ After deletion of 5 subjects, as discussed in text, $F(1,26)=9.54, p<.01$. DLPFC, dorsolateral prefrontal cortex; ACG, anterior cingulate gyrus; IPL, inferior parietal lobule; PCG, postcentral gyrus; FWM and PWM, front and parietal white matter; HF, hippocampal formation; Lat V, lateral ventricles. ${ }^{*} p<.05 . \quad \dagger p<.01 . \quad \ddagger p<.001$.

Table 3

Effects of Age and Sex on Selected Cerebral Structures After Mutual Adjustment and Adjustment for Skull Size

\begin{tabular}{|c|c|c|}
\hline Structure & $F$ for Age & $F$ for Sex \\
\hline$\overline{D^{\prime} P F C} \bar{C}^{1,2}$ & .04 & .02 \\
\hline ACG & .36 & .99 \\
\hline IPL & 3.02 & 1.37 \\
\hline PCG & 3.06 & .00 \\
\hline$V C^{1}$ & 1.71 & $4.51^{*}$ \\
\hline FWM & .39 & .43 \\
\hline PWM & .24 & .01 \\
\hline $\mathrm{HF}$ & .66 & $4.27^{*}$ \\
\hline Lat $\mathrm{V}$ & $16.26 \dagger$ & 2.86 \\
\hline
\end{tabular}

${ }^{1}$ Full model. ${ }^{2}$ After deletion of 5 subjects as discussed in the text, $F(1,26)=10.20$ and $F(1,26)=1.00$, both $p s<.01$, for age and sex, respectively. DLPFC, dorsolateral prefrontal cortex; ACG, anterior cingulate gyrus; IPL, inferior parietal lobule; PCG, postcentral gyrus; VC, visual cortex; FWM and PWM, frontal and parietal white matter; $\mathrm{HF}$, hippocampal formation; Lat $\mathrm{V}$, lateral ventricles. ${ }^{*} p<.05$. $\dagger p<.001$.

This interaction stemmed from the fact that for 4 elderly males $(65,65,76$, and 77 years old) with the smallest skull size, and a young female (28 years old) with the largest skull, the relationship between DLPFC volume and age was reversed-a strong positive correlation was observed. After these 5 subjects were removed from the sample, the model was successfully reduced, and a significant age-related decline in the DLPFC was observed.

\section{Sex Differences}

Several significant sex differences were observed. After adjustment for skull size and age, males still evidenced larger visual areas than did females, whereas the females had larger hippocampal formations.

The model for the visual areas contained a significant age $\times$ sex interaction; males showed virtually no agerelated decline $(r=-.18$, n.s.), whereas females evi- denced a trend for age-dependent shrinkage $(r=-.52$, $p<.08$ ).

To summarize, in Study 1, we replicated a benchmark finding of cerebral aging: cerebral ventriculomegaly and leftward asymmetry of the lateral ventricles (DeCarli, Kaye, Horwitz, \& Rapoport, 1990; Freedman et al., 1984). More important, however, are the results pointing to selective age-related cerebral atrophy. The prefrontal association areas appeared to be more vulnerable to aging than the primary somatosensory cortex and the primary and secondary visual areas. Unexpectedly, we observed no trend for age-related shrinkage of the hippocampal formation. A tendency of the cerebral cortex to be larger on the right than on the left was observed as well.

Inasmuch as zero-order correlations appear to support the hypothesized age trends, adjustment for skull size and sex attenuated virtually all of them. On the one hand, these corrections were necessary to avoid confounding body size and brain size differences. On the other hand, the significant age-skull size correlation could have been a spurious sample-specific phenomenon that had masked true agerelated trends. In an attempt to replicate the findings obtained in Study 1, we repeated some of the morphometric measurements on a larger sample. Study 2 was a retrospective evaluation of normal MRI scans that were obtained from the patients with neurological complaints who underwent the procedure as a part of diagnostic work-up.

\section{STUDY 2}

\section{Method}

Subjects

The MRI images of 54 patients were acquired at the Diagnostic Imaging Center at Baptist Memorial Hospital in Memphis. The referrals were based on a variety of neurological complaints, such as headaches (23\%), tingling and burning sensation in the limbs and face $(14.6 \%)$, dizziness and nausea $(14.5 \%)$, poor memory $(8 \%)$, back and neck pain ( $8 \%)$, syncopy $(6.3 \%)$, blurred vision $(4 \%)$, transient paralysis $(4 \%)$, and hearing difficulties $(2 \%)$. About $15 \%$ of the patients were referred to rule out multiple sclerosis. Some patients presented with multiple complaints, but no complaint was of a psychiatric nature. All scans were reviewed by a neuroradiologist and revealed no pathological findings. This sample included 18 males and 36 females. The mean age was 41.2 years $(S D=14.54)$, with a range of 18-75 years. Because of the archival nature of this study, no data on formal education or handedness were available.

\section{Apparatus and Procedure}

Imaging was performed on a Signa 1.5T scanner (General Electric, Milwaukee) at the Diagnostic Imaging Center. Although many of the patients underwent multiple imaging sequences required for their specific diagnostic questions, only two of these sequences were selected for analysis. The sagittal spin-echo series was acquired at $\mathrm{TE}=20 \mathrm{msec}, \mathrm{TR}=600-800 \mathrm{msec}, \mathrm{NEX}=1-2, t=5 \mathrm{~mm}$, and $\mathrm{I}=2.5 \mathrm{~mm}$. The acquisition parameters of the double-echo coronal sequence were $\mathrm{TE}=30$ or $80 \mathrm{msec}$, $\mathrm{TR}=2,300$ $2,800 \mathrm{msec}$, and $t=5 \mathrm{~mm}$, with I varying among the scans. The interslice gaps were $1 \mathrm{~mm}$ (14\% of the scans), $1.5 \mathrm{~mm}(45 \%), 2 \mathrm{~mm}$ $(33 \%)$, or $2.5 \mathrm{~mm}(8 \%)$. Only proton-weighted images of the double-echo sequence were used in this study. All images were filmed with window and level settings in a narrow range ( $W=$ 1,050-1,175; $L=140-230$ ). 
Table 4

Correlations Among ROI Areas and Age

\begin{tabular}{lccccccccc}
\hline Structure & DLPFC & ACG & STG & SSC & VC & PHG & HF & FWM & Age \\
\hline Skull & $.27^{*}$ & .19 & .22 & .13 & .18 & .06 & -.17 & .37 & .03 \\
DLPFC & & .22 & $.39 \dagger$ & .11 & .18 & $.32^{*}$ & .22 & $.62 \ddagger$ & $-.58 \ddagger$ \\
ACG & & & $.37 \dagger$ & .04 & .14 & -.10 & .27 & .07 & -.15 \\
STG & & & & $.52 \ddagger$ & .08 & $.30^{*}$ & .19 & .20 & -.23 \\
SSC & & & & & .11 & .22 & -.14 & -.05 & .02 \\
VC & & & & & & -.17 & -.42 & .07 & -.14 \\
PHG & & & & & & & -.17 & -.03 & -.04 \\
HF & & & & & & & & .16 & -.23 \\
FWM & & & & & & & & & $-.36 \dagger$ \\
\hline
\end{tabular}

Note $-N=54$ for all measures except PHG $(N=42)$, and HF $(N=40)$. DLPFC, dorsolateral prefrontal cortex; ACG, anterior cingulate gyrus; STG, superior temporal gyrus; SSC, somatosensory cortex; VC, visual cortex; PHG, parahippocampal gyrus; HF, hippocampal formation; FWM, frontal white matter. ${ }^{*} p<.05 . \quad \nmid p<.01$. $\ddagger p<.001$.

\section{Computerized Image Analysis}

Although the image analysis procedures in Study 2 and Study 1 were identical, the lists of ROIs differed. In Study 2, the IPL could not be demarcated reliably on many scans. Its volume, as well as the volume of parietal white matter and the ventricles, was not estimated. However, the cortices of the parahippocampal and the superior temporal gyri were measured. In addition, the average cross-sectional area of the DLPFC was estimated from two slices (instead of one), and the hippocampal formation was measured on three consecutive slices (instead of two). The parahippocampal gyrus was not visualized on 12 scans, and the hippocampal formation was not measured on 2 additional scans. Due to the fact that the scans differ in the interslice gaps, the volumes of the ROIs were not estimated; average cross-sectional areas were computed instead. The rules for tracing the ROIs added in Study 2 are outlined below.

\section{Superior Temporal Gyrus}

The rostral border of the superior temporal gyrus was identified on the rostral-most coronal slice in which the temporal stem could be visualized. The superior temporal gyrus was defined as the cortex ventral to the Sylvian fissure and dorsal to the superior temporal sulcus. The caudal border of the superior temporal gyrus was the slice through the splenium of the corpus callosum at which the superior temporal gyrus became indistinguishable from the IPL.

\section{Parahippocampal Gyrus}

This structure was measured only on the same three slices as was the hippocampal formation. The parahippocampal gyrus consisted of the medial gray matter of the temporal lobes, extending dorsally from the hippocampus to the parahippocampal sulcus ventrally. Because there was no distinct demarcation of the upper boundary of the parahippocampal gyrus, it was decided that only the medial surface would be measured. This upper boundary was drawn as the structure (continuous with the subiculum) turned laterally. The ventral boundary was demarcated by a straight line drawn through the parahippocampal sulcus.

Reliability analysis of the ROI measures yielded intraclass correlations virtually identical to those obtained in Study 1 . The reliability of the superior temporal and parahippocampal gyrus measures for two operators and 6 randomly selected subjects were .93 and .91 , respectively.

\section{Results and Discussion}

The zero-order correlations among the brain measures and age are presented in Table 4. Age, sex, and lateral differences in the size of the selected brain structures adjusted for skull size were examined in the framework of general linear models as in Study 1. The deviation scores were used for skull size and age. Full models (with all interactions) were tested first. If the assumption of regression homogeneity was satisfied, the reduced models were analyzed. The results of the analyses are presented in Tables 5 (descriptive statistics and hemispheric differences) and 6 (age and sex effects).

\section{Hemispheric Differences}

Several instances of hemispheric structural asymmetry were observed in the brains examined in this sample. The direction of asymmetry for all ROIs was consistent with the results of Study 1: leftward asymmetry was observed in the prefrontal white matter, whereas the cortical gray matter exhibited a rightward asymmetry trend. The latter trend, however, was very weak and reached significance only for the superior temporal gyrus, for which a significant sex $\times$ side interaction $[F(1,46)=7.56, p<$ $.01]$ was found. This interaction was due to the fact that

Table 5

Average Areas of Selected Cerebral Structures by Hemisphere After Adjustment for Age, Sex, and Skull Size ${ }^{1}$

\begin{tabular}{|c|c|c|c|c|c|}
\hline \multirow[b]{3}{*}{ Structure } & \multicolumn{4}{|c|}{ Size } & \multirow{2}{*}{$\begin{array}{c}\text { Left-Right } \\
\text { Comparison }^{2} \\
\end{array}$} \\
\hline & \multicolumn{2}{|c|}{ Left } & \multicolumn{2}{|c|}{ Right } & \\
\hline & $M$ & $S D$ & $M$ & $S D$ & $\bar{F}$ \\
\hline $\operatorname{DLPFC}^{3}\left(\mathrm{~cm}^{2}\right)$ & 6.33 & .86 & 6.38 & .76 & .19 \\
\hline $\mathrm{ACG}^{3}\left(\mathrm{~cm}^{2}\right)$ & 1.86 & .30 & 1.95 & .29 & .72 \\
\hline $\mathrm{STG}^{4}\left(\mathrm{~cm}^{2}\right)$ & 2.03 & .29 & 2.07 & .25 & $9.87 *$ \\
\hline PHG $\left(\mathrm{cm}^{2}\right)$ & .46 & .09 & .46 & .08 & .11 \\
\hline PCG $\left(\mathrm{cm}^{2}\right)$ & 2.98 & .44 & 3.07 & .59 & .50 \\
\hline FWM $\left(\mathrm{cm}^{2}\right)$ & 9.97 & 1.76 & 8.98 & 1.63 & $17.95 \dagger$ \\
\hline \multirow[t]{2}{*}{$\mathrm{HF}\left(\mathrm{cm}^{2}\right)$} & .47 & .06 & .47 & .06 & .03 \\
\hline & & \multicolumn{2}{|c|}{$M$} & \multicolumn{2}{|c|}{$S D$} \\
\hline \multirow{2}{*}{\multicolumn{2}{|c|}{$\begin{array}{l}\text { Midskull area }\left(\mathrm{cm}^{2}\right) \\
\text { Visual areas }\left(\mathrm{cm}^{2}\right)\end{array}$}} & \multirow{2}{*}{\multicolumn{2}{|c|}{$\begin{array}{r}166.43 \\
10.50\end{array}$}} & \multirow{2}{*}{$\begin{array}{r}12.29 \\
2.44\end{array}$} & \\
\hline & & & & & \\
\hline
\end{tabular}
${ }^{1} N=54$ for all measures except PHG $(N=42)$ and HF $(N=$ 40). ${ }^{2}$ After adjustment for skull size, age, and sex. ${ }^{3}$ Full model, all interactions included. ${ }^{4} \mathrm{~A}$ significant side $\times$ sex interaction, discussed in the text. DLPFC, dorsolateral prefrontal cortex; ACG, anterior cingulate gyrus; STG, superior temporal gyrus; PHG, parahippocampal gyrus; PCG, postcentral gyrus; FWM, frontal white matter; HF, hippocampal formation. ${ }^{*} p<.01 . \quad \dagger p<.001$. 
Table 6

Effects of Age and Sex on Selected Cerebral Structures After Mutual Adjustment and Adjustment for Skull Size ${ }^{1}$

\begin{tabular}{lcc}
\hline Structure & $F$ for Age & $F$ for Sex \\
\hline DLPFC $^{2}$ & $29.01^{*}$ & .00 \\
ACG $^{2}$ & .01 & 2.02 \\
STG & 3.08 & .07 \\
PHG & .03 & .69 \\
PCG & .01 & .00 \\
FWM & $8.88^{*}$ & .14 \\
HF & 1.22 & .54 \\
VC & 1.83 & 2.56 \\
\hline
\end{tabular}

${ }^{1} N=54$ for all measures except PHG $(N=42)$, and HF $(N=40)$. ${ }^{2}$ Full model, all interactions included. DLPFC, dorsolateral prefrontal cortex; ACG, anterior cingulate contex; STG, superior temporal gyrus; PHG, parahippocampal gyrus; PCG, postcentral gyrus; FWM, frontal white matter; HF, hippocampal formation; VC, visual cortex. $\quad{ }^{*} p<.005$.

only men showed significant rightward asymmetry $(2.13$ vs. $1.98 \mathrm{~cm}^{2}$ ), whereas among the women, the right and the left cortices were virtually even $\left(2.05\right.$ and $\left.2.03 \mathrm{~cm}^{2}\right)$.

\section{Age and Sex Effects}

After adjustment for head size and sex, significant agerelated shrinkage was observed in the DLPFC and in prefrontal white matter. As indicated by a significant age $\times$ sex interaction $[F(1,46)=7.17, p<.01]$, the slopes of age-related decreases in the DLPFC volume differed between the sexes. Men showed a steeper age-related decline of the prefrontal cortex area $(r=-.78, p<.001$, compared to $r=-.43, p<.05$, for women).

A significant skull size $\times$ sex interaction was found in the full model for the anterior cingulate $[F(1,46)=4.89$, $p<.05]$ gyrus. This interaction reflected sex differences in correlations between the skull and the ROI size ( $r=$ $.35, p<.05$, for females, and $r=-.29$, n.s., for males). Because of this violation of the homogeneity of regression assumption, skull size could not be used as a covariate in the reduced model.

\section{GENERAL DISCUSSION}

The results of the two studies reported here support the notion of selective aging of the human cerebral cortex. Although in Study 1 age trends were attenuated by correction for skull size, the pattern of zero-order correlations between age and the cortical ROI measures was virtually identical across the two samples (Figure 2).

The composition of the samples varied considerably with respect to subjects' health status and demographic characteristics; the parameters of MRI scanners and acquisition procedures at two sites were dissimilar as well. All these factors may be expected to introduce irrelevant variability into brain measures obtained in two studies. In addition, because of the relatively small sample size $(N=29)$, the confidence limits of the correlation coefficients obtained in Study 1 were rather broad, and the chances of replicating these correlations in Study 2 were poor. Thus, replication of the pattern of correlations despite the aforementioned adverse factors is evidence of the robustness of the findings.

The results suggest that in the aging brain, the polymodal association cortex areas are affected to a greater extent than are the primary and secondary sensory cortices. Although the evidence of age-related pathoclysis in the human cerebral cortex can be found in postmortem studies (Kemper, 1984), it has not been, to the best of our knowledge, observed in neuroanatomical studies in vivo. A recent study of brain glucose metabolism in young and elderly women provides convergent evidence of selective vulnerability of the frontal and parietal association cortices (Azari et al., 1992). The emerging picture of
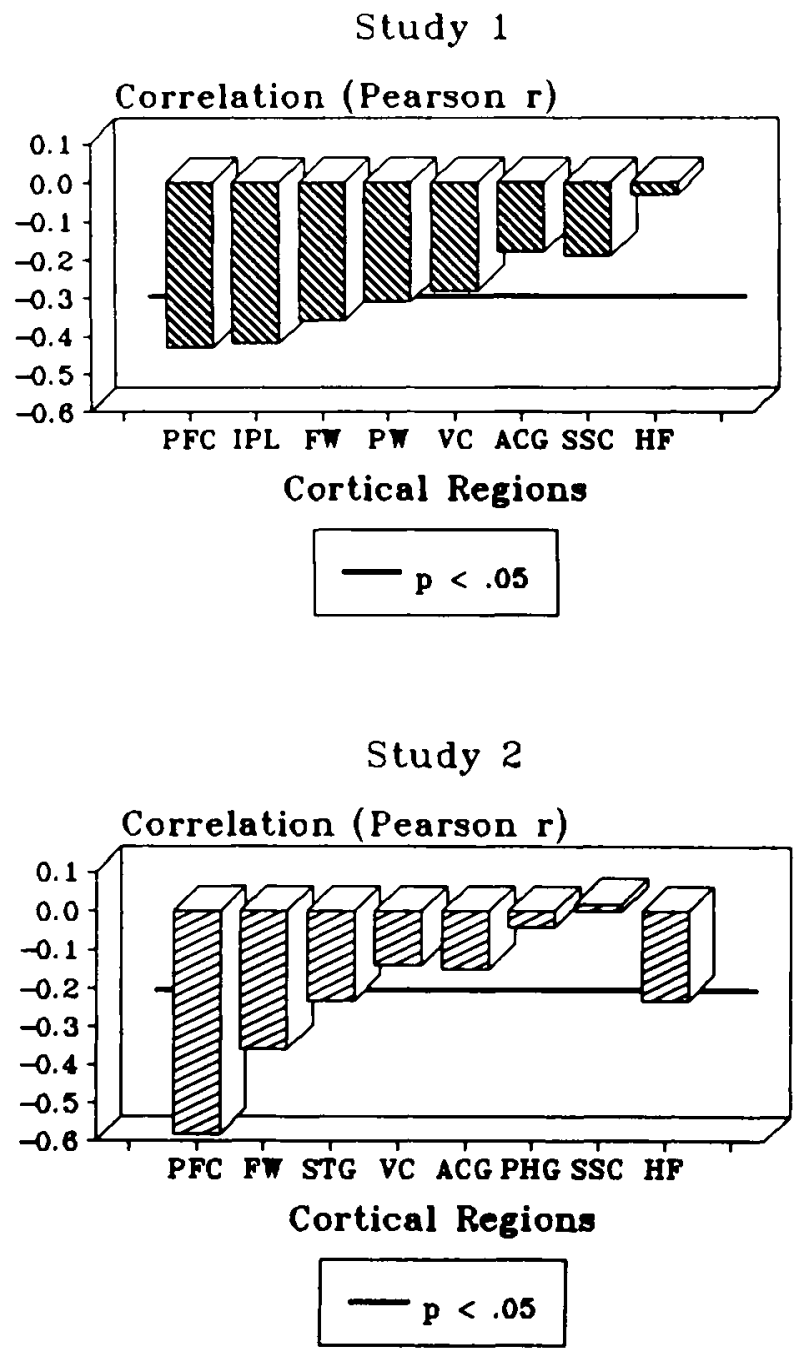

Figure 2. Patterns of correlations between cortical structures and age observed in two samples. PFC, prefrontal cortex; IPL, inferior parietal lobule; FW, frontal white matter; PW, parietal white matter; VC, visual areas; ACG, anterior cingulate gyrus; SSC, somatosensory cortex; HF, hippocampal formation; STG, superior temporal gyrus; PHG, parahippocampal gyrus. 
differential brain aging contradicts the notion of global age-related degeneration fostered by widely replicated nonspecific findings such as age-related ventriculomegaly (Freedman et al., 1984). If the brain regions indeed age at uneven paces, the causes and mechanisms of such selective aging are unknown. A number of neurochemical, developmental, and phylogenetic factors have been proposed to explain the topography of age-related changes in the cerebral cortex, but to date no unified model has emerged.

Several theories of brain aging suggest that the loss of cortical neurons may be mediated by age-related neurochemical changes. One of these theories is focused on age-related declines in catecholaminergic function (Goldman-Rakic, 1987), which may stem from age-related atrophy of the subcortical sources of catecholamines (McGeer et al., 1990). Indeed, the prefrontal cortex, one of the major recipients of subcortical dopaminergic and noradrenergic projections (Fallon \& Loughlin, 1987), shows significant age-related declines, whereas the primary visual cortex - a region with sparse catecholaminergic innervation (Levitt, Rakic, \& Goldman-Rakic, 1984) -is spared by aging. The regional distribution of age-related cortical shrinkage observed in Studies 1 and 2 is consistent with predictions of the catecholaminergic model of aging.

A model of brain aging based on the central cholinergic pathways (Bartus, Dean, Pontecorvo, \& Flicker, 1985) predicts selective age-related deterioration of structures that receive dense cholinergic projections from the nucleus basalis of Meynert-the hippocampus, the parahippocampal and cingulate gyri, and the insula (Mesulam, Rosen, $\&$ Mufson, 1984). We have measured most of these structures, and we have observed age-related shrinkage in none. Thus, the findings reported here do not support the predictions of the cholinergic model of brain aging. Given the relatively small sample size, the negative conclusions of Studies 1 and 2 should be treated with caution. It is quite possible that with additional statistical power, decline of the cholinergic structures in the normally aging brain can be demonstrated in future studies.

Finally, according to the excitotoxic model, brain aging is a result of cumulative damage inflicted on areas that are rich in excitatory amino acid receptors. Neurons in such areas-pyramidal neurons in the hippocampus, for example-are highly susceptible to the cumulative effects of hypoxia and ischemia that may occur even in ostensibly healthy elderly (Cotman \& Monaghan, 1987). Surprisingly, there are virtually no data on age-related and regional differences in cortical excitatory amino acid activity (Morgan \& May, 1990). Thus, the results reported in this article cannot be related to the excitotoxic model of cortical aging.

The preceding neurochemical models of brain aging are not mutually exclusive. Rather, they may characterize complementary pathoclytic aspects of cortical aging, and they may interact in creating localized pathoclytic areas (Advokat \& Pellegrin, 1992). Several patterns of cortical damage may be observed in the brains of the aging population. Yet, in each individual, only one of these pat- terns may predominate. Although the configuration of cortical aging reported here agrees with the catecholaminergic deafferentation model, other samples may yield different topographic distributions of cortical shrinkage.

Age-related decline in the volume of the white matter was observed in the prefrontal regions in both samples. This finding appears to contradict the previously reported stability of gross white-matter volume across the adult life span (Jernigan et al., 1990; Lim et al., 1992). However, in one of these studies (Jernigan et al., 1990), regional white-matter volume was not measured, whereas in the other (Lim et al., 1992), in spite of the lack of age differences in total hemispheric white matter, in the elderly, the prefrontal white-matter volume was reduced by 1.3 standard deviations in relation to that of the young subjects. Whether aging is associated with selective changes in the white matter is a question to be answered by future studies concentrating on finer and more comprehensive regional analysis than has been attempted to date.

There is one notable discrepancy between the regional distribution of vulnerability to aging observed in our studies and the previously reported pattern of selective brain aging. In both samples, we found no significant agerelated decline in the volume of the hippocampal formation, although a trend was observed in Study 2. It is possible that despite the high reliability of the hippocampal formation measures, the validity of the volume estimates was limited. Two (Study 1) or even three (Study 2) slices may not be sufficient for obtaining a stable volume estimate; eight systematically sampled slices per structure is the recommended minimum (Mayhew \& Olsen, 1991). Indeed, in other samples, more stable estimates of hippocampal volume based on greater numbers of slices have revealed moderate age-related atrophy (Coffey et al., 1992).

In contradiction to postmortem findings (Arriagada \& Hyman, 1990; Kemper, 1984), no age-related changes were observed in the parahippocampal gyrus. We can merely speculate that in normal aging, only specific cortical laminae of that region may be affected as Alzheimer's disease (A. R. Damasio, Van Hoesen, \& Hyman, 1990). Such specific laminar atrophy would be difficult to detect with MRI morphometry. Interestingly, the hippocampal formation and parahipocampal gyrus pathology may be independent, for the latter shows no signs of shrinkage in amnestic patients with demonstrable hippocampal degeneration (Hopkins \& Kesner, 1993; Squire, Amaral, \& Press, 1990).

Several methodological limitations in the present studies should be taken into account in any interpretion of the results. In addition to the stated limitations of statistical power and subject selection, an important issue of the neuroanatomical interpretation of the MRI morphometry should not be overlooked. The observed shrinkage of the cortical ribbon may reflect a loss of neuronal bodies, glia, or neuropil and its specific components. In light of the evidence of age-related neuronal loss, neuronal shrinkage, and dendritic atrophy (Coleman \& Flood, 1987), and the reported lack of decline in glial population (Devaney \& 
Johnson, 1980; Peters, Josephson, \& Vincent, 1991), it is most likely that neuronal and not glial changes underlie gray-matter declines in the aging brain. Decreases in the white-matter volume are likely to reflect the thinning of the myelin sheath. Indeed, age-related alterations in proton relaxation times support that view (Jernigan et al., 1991; Raz et al., 1990; Wahlund et al., 1990). There is no evidence, however, that changes in molecular behavior of white-matter water are related to age-related tissue loss (Jernigan et al., 1991).

To summarize, in vivo MRI morphometry revealed a pattern of differential aging of the cerebral cortex. The observed pathoclytic configuration of age-related differences is consistent with the catecholaminergic model of brain aging. The ultimate question, however, is, what are the cognitive and behavioral consequences of observed cortical pathoclysis? Normal aging may provide just the right range of variability in structure and function to allow exploration of the relations between the two.

\section{REFERENCES}

Advokat, C., \& Pellegrin, A. I. (1992). Excitatory amino acids and memory: Evidence from research on Alzheimer's disease and behavioral pharmacology. Neuroscience \& Biobehavioral Reviews, 16, 13-24.

ARriagada, P. V., \& Hyman, B. T. (1990). Entorhinal cortex is an early site of neurofibrillary tangle pathology in nondemented elderly. Society for Neuroscience Abstracts, 16, 1265.

azari, N. P., Rapoport, S. I., Salerno, J. A., Grady, C. L., Gonzales-Aviles, A., Schapiro, M. B., \& Horwitz, B. (1992). Interregional correlations of resting cerebral glucose metabolism in old and young women. Brain Research, 589, 279-290.

Bartus, R. T., Dean, R. L., Pontecorvo, M. J., Flicker, C. (1985). The cholinergic hypothesis: A historical overview, current perspective, and future directions. In D. S. Olton, E. Gamzu, \& S. Corkin (Eds.), Memory dysfunctions: An integration of animal and human research from preclinical and clinical perspectives (Annals of New York Academy of Sciences, Vol. 444, pp. 332-358). New York: New York Academy of Sciences.

Brody, H. (1973). Aging in the vertebrate brain. In M. Rockstein (Ed.), Development and aging in the nervous system (pp. 121-134). New York: Academic Press.

Coffey, C. E., Wilkinson, W. E., Parashos, I. A., Soady, S. A. R., Sullivan, R. J., Patterson, L. J., Figiel, G. S., Webb, M. C., Spritzer, C. E., \& DJANG, W. T. (1992). Quantitative cerebral anatomy of the aging human brain: A cross-sectional study using magnetic resonance imaging. Neurology, 42, 527-536.

Coleman, P. D., \& Flood, D. G. (1987). Neuron numbers and dendritic extent in normal aging and Alzheimer's disease. Neurobiology of Aging, 8, 521-545.

Cotman, C. W., Monaghan, D. T. (1987). Chemistry and anatomy of excitatory amino acid systerns. In H. Y. Meltzer (Ed.), Psychopharmacology: The third generation of progress (pp. 197-210). New York: Raven Press.

Damasio, A. R., Van Hoesen, G. W., \& Hyan, B. T. (1990). Reflections on the selectivity of neuropathological changes in Alzheimer's disease. In M. F. Schwartz (Ed.), Modular deficits in Alzheimer-type Dementia (pp. 83-100). Cambridge, MA: MIT Press.

Damasio, H., \&amasio, A. R. (1989). Lesion analysis in neuropsychology. New York: Oxford University Press.

Daniels, D. L., Naughton, V. M., \& Naidich, T. P. (1987). Cranial and spinal MRI: An atlas and guide. New York: Raven Press.

DeArmond, S. J., Fusco, M. M., \& DeweX, M. M. (1976). Struc- ture of human brain: A photographic atlas. New York: Oxford University Press.

DeCarli, C., Kaye, J. A., Horwitz, B., \& Rapoport, S. I. (1990). Critical analysis of the use of computer-assisted transverse axial tomography to study human brain in aging and dementia of the Alzheimer type. Neurology, 40, 872-883.

Devaney, K. O., Johnson, H. A. (1980). Neuronal loss in the aging visual cortex of man. Journal of Gerontology, 35, 836-841.

DrAyer, B. P. (1988). Imaging of the aging brain: Part 1. Normal findings. Radiology, 166, 785-796.

Fallon, J. H., Loughlin, S. E. (1987). Monoamine innervation of cerebral cortex and a theory of the role of monoamines in cerebral cortex and basal ganglia. In E. Jones \& A. Peters (Eds.), Cerebral cortex: Vol 6. Further aspects of cortical functions, including hippocampus (pp. 41-128). New York: Plenum.

Finlay, J., \& Richardson, A. (1990). An investigation into the age of cessation of endocranial growth using the techniques of logetronic copying and photosubtraction. Annals of Human Biology, 17, 289-296.

Folstein, M. F., Folstein, S. E., McHugh, P. R. (1975). Minimental state: A practical method for grading the cognitive state of patients for the clinician. Journal of Psychiatric Research, 12, 189-198.

Freedman, M., Knoefel, J., Naeser, M., Levine, H. (1984). Computerized axial tomography in aging. In M. L. Albert (Ed.), Clinical neurology of aging (pp. 139-148). New York: Oxford University Press.

Goldman-RAKIC, P. S. (1987). Circuitry of primate prefrontal cortex and regulation of behavior by representational memory. In V. Mountcastle \& F. Plum (Eds.), Handbook of physiology: The nervous system: Vol. 5 (pp. 373-417). Baltimore, MD: American Physiological Society.

HAUG, H. (1985). Are neurons of the human cerebral cortex really lost during aging? A morphometric examination. In J. Tarber \& W. H. Gispen (Eds.), Senile dementia of Alzheimer type (pp. 150-163). Berlin: Springer-Verlag

HoPKINS, R. O., KESNER, R. P. (1993). Data-based and knowledgebased memory for temporal order information in subjects with hypoxic brain injury. Manuscript submitted for publication.

Jernigan, T. L., Archibald, S. L., Berhow, M. T., Sowell, E. R., Foster, D. S., \& Hesselunk, J. R. (1991). Cerebral structure on MRI: Part 1. Localization of age-related changes. Biological Psychiatry, 29, 55-67.

Jernigan, T. L., Press, G. A., \& Hesselink, J. R. (1990). Methods for measuring brain morphologic features on magnetic resonance images: Validation and normal aging. Archives of Neurology, 45, 404-408.

KatZman, R., Terry, R. (1983). Normal aging of the nervous system. In R. Katzman \& R. Terry (Eds.), The neurology of aging (pp. 15-50). Philadelphia: F. A. Davis.

KEMPER, T. (1984). Neuroanatomical and neuropathological changes in normal aging and in dementia. In M. L. Albert (Ed.), Clinical neurology of aging (pp. 9-52). New York: Oxford University Press

LevitT, P., Rakic, P., \& Goldman-Rakic, P. S. (1984). Regionspecific distribution of catecholamine afferents in primate cerebral cortex: A fluorescence histochemical analysis. Journal of Comparative Neurology, $225,1-14$.

Lim, K., Zipurski, R. B., Murphy, G. M., \& Pfefferbaum, A. (1990). In vivo quantification of the limbic system using MRI: Effects of normal aging. Psychiatry Research: Neuroimaging, 35, 15-26.

Lim, K. Zipurski, R. B., Watts, M. C., \& Pfefferbaum, A. (1992). Decreased gray matter in normal aging: An in vivo magnetic resonance study. Journal of Gerontology: Biological Sciences, 47, 826-830.

MANI, R. B., LoHR, J. B., \& JESTE, D. V. (1986). Hippocampal pyramidal cells in the human: A quantitative study of neuronal loss in sectors CA1 through CA4. Experimental Neurology, 94, 29-40.

MAYHEW, T. M., \& OLSEN, D. R. (1991). Magnetic resonance imaging (MRI) and model-free estimates of brain volume determined using the Cavalieri principle. Journal of Anatomy, 178, 133-144

McGeer, P. L., McGeer, E. G., Akiyama, H., Itagaki, S., HarROP, R., \& PEPPARD, R. (1990). Neuronal degeneration and memory loss in Alzheimer's disease and aging. In J. Eccles \& O. Creutzfeldt 
(Eds.), The principles of design and operation of the brain (pp. 410431). Berlin: Springer-Verlag.

Mesulam, M.-M., Rosen, A. D., \& Mufson, E. J. (1984). Regional variations in cortical cholinergic innervation chemoarchitectonics of acetylcholinesterase containing fibers in the macaque brain. Brain Research, 311, 254-258.

Miller, A. K. H., Alston, R. L., \& Corsellis, J. A. N. (1980). Variation with age in the volumes of gray and white matter in the cerebral hemispheres of man: Measurements with an image analyser. Neuropathology \& Applied Neurobiology, 6, 119-132.

Montemurro, D. G., \& BRUni, J. E. (1980). The human brain in dissection. New York: Oxford University Press.

Morgan, D. G., \& MAY, P. C. (1990). Age-related changes in synaptic neurochemistry. In E. L. Schneider \& J. W. Rowe (Eds.), Handbook of the biology of aging (3rd ed., pp. 219-254). San Diego, CA: Academic Press.

Nieuwenhuys, R., Voogd, J., \& Huiszen, C. (1988). Central nervous system: A synopsis and atlas. Berlin: Springer-Verlag.

Peres, N. S., Kane, W. C., \& Aronson, S. M. (1973). Central nervous system findings in a tenth decade autopsy population. In D. H. Ford (Ed.), Neurobiological aspects of maturation and aging (Progress in Brain Research, Vol. 40, pp. 473-483). Amsterdam: Elsevier.

Peters, A., Josephson, K., \& Vincent, S. L. (1991). Effects of aging on the neuroglial cells and pericytes within area 17 of the rhesus monkey cerebral cortex. Anatomical Record, 229, 384-398.

Raz, N., Millman, D., \& Sarpel, G. (1990). Cerebral correlates of cognitive aging: Gray-white-matter differentiation in the medial temporal lobes, and fluid versus crystallized abilities. Psychobiology, 18, 475-481.

Raz, N., Torres, I. J., Spencer, W. D., Millman, D., Baertschi, J. C., \& SARPEL, G. (in press). Neuroanatomical correlates of agesensitive and age-invariant cognitive abilities: An in vivo MRI investigation. Intelligence.

SCHEIBEL, A. B. (1981). The gerohistology of the aging human forebrain: Some structuro-functional considerations. In $S$. J. Enna, T. Samorajski, \& B. Beer (Eds.), Brain neurotransmitters and recep- tors in aging and age-related disorders (pp. 31-41). New York: Raven Press.

Schwartz, M., Creasey, H., Grady, C. M., Deleo, J. A., Frederickson, H. A., Cutler, N. R., \& Rapoport, S. I. (1985). Computerized tomographic analysis of brain morphometrics in 30 healthy men, aged 21 to 81 years. Annals of Neurology, 17, 146-153.

Shrout, P. E., \& FLEISs, J. L. (1979). Intraclass correlations: Uses in assessing raters reliability. Psychological Bulletin, 86, 420-428.

SKULLERUD, K. (1985). Variations in the size of the human brain: Influence of age, sex, body length, body mass index, alcoholism, Alzheimer changes, and cerebral atherosclerosis. Acta Neurologica Scandinavica, 71, 1-94.

Squire, L. R., Amaral, D. G., \& Press, G. (1990). Magnetic resonance imaging of the hippocampal formation and mamillary nuclei distinguishes medial temporal lobe and diencephalic amnesia. Journal of Neuroscience, 10, 3106-3117.

Terry, R. D., DeTeresa, R., \& Hansen, L. A. (1987). Neocortical cell counts in normal human adult aging. Annals of Neurology, 21, 530-539.

Tigges, J., Herndon, J. G., \& Peters, A. (1990). Neuronal population of area 4 during the life span of the rhesus monkey. Neurobiology of Aging, 11, 201-208.

Vincent, S. L., Peters, A., \& Tigges, J. (1989). Effects of aging on the neurons within the area 17 of the rhesus monkey cerebral cortex. Anatomical Record, 223, 329-341.

VoGT, C., \& VoGT, O. (1951). Importance of neuroanatomy in the field of neuropathology. Neurology, 1, 205-218.

Wahlund, L.-O., Agartz, I., Almovist, O., Basun, H., ForsSell, L., SAAF, J., \& WetTerberG, L. (1990). The brain in healthy aged individuals: MR imaging. Radiology, 174, 675-679.

White, L. R., Cartwright, W. S., Cornoni-Huntley, J., \& Brock, D. B. (1986). Geriatric epidemiology. Annual Review of Gerontology \& Geriatrics, 6, 215-311.

(Manuscript received November 16, 1992; revision accepted for publication January 25, 1993.) 